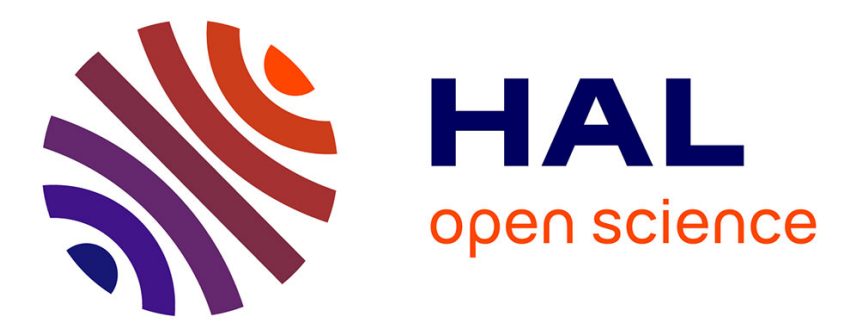

\title{
Optical and structural characterization of thin films containing metallophthalocyanine chlorides
}

Anna Zawadzka, Asli Karakas, P. Plóciennik, J. Szatkowski, Z. Łukasiak, A. Kapceoglu, Y. Ceylan, Bouchta Sahraoui

\section{- To cite this version:}

Anna Zawadzka, Asli Karakas, P. Plóciennik, J. Szatkowski, Z. Łukasiak, et al.. Optical and structural characterization of thin films containing metallophthalocyanine chlorides. Dyes and Pigments, 2015, 112, pp.116-126. 10.1016/j.dyepig.2014.06.029 . hal-01391696

\section{HAL Id: hal-01391696 \\ https://hal.science/hal-01391696}

Submitted on 20 Sep 2021

HAL is a multi-disciplinary open access archive for the deposit and dissemination of scientific research documents, whether they are published or not. The documents may come from teaching and research institutions in France or abroad, or from public or private research centers.
L'archive ouverte pluridisciplinaire HAL, est destinée au dépôt et à la diffusion de documents scientifiques de niveau recherche, publiés ou non, émanant des établissements d'enseignement et de recherche français ou étrangers, des laboratoires publics ou privés.

\section{(c)(1)}

Distributed under a Creative Commons Attribution| 4.0 International License 


\title{
Optical and structural characterization of thin films containing metallophthalocyanine chlorides
}

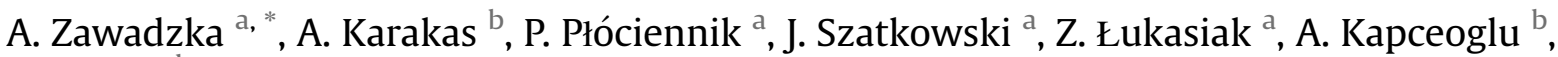 \\ Y. Ceylan ${ }^{\text {b }}$, B. Sahraoui ${ }^{\mathrm{c}}$ \\ ${ }^{a}$ Institute of Physics, Faculty of Physics, Astronomy and Informatics, Nicolaus Copernicus University, Grudziadzka 5, 87-100 Torun, Poland \\ ${ }^{\mathrm{b}}$ Department of Physics, Faculty of Sciences, Selcuk University Campus, Konya, Turkey \\ ${ }^{\mathrm{c}}$ LUNAM Université, Université d'Angers, CNRS UMR 6200, Laboratoire MOLTECH-Anjou, 2 bd Lavoisier, 49045 Angers Cedex, France
}

\begin{abstract}
The structural and optical investigation of thin films containing aluminum and gallium phthalocyanine chlorides is presented. The films were fabricated by Physical Vapor Deposition technique onto quartz substrates and annealed after fabrication in an ambient atmosphere for $24 \mathrm{~h}$ at the temperature equal to $150{ }^{\circ} \mathrm{C}$ or $250^{\circ} \mathrm{C}$. The experimental results and theoretical calculation of the Third Harmonic Generation process are reported. The third order nonlinear optical properties are expected and can be more or less accurately predicted due to the assembly of the molecules and theoretical calculations of the frequencydependent dipole polarizabilities, third hyperpolarizabilities, third order susceptibilities, frontier and second frontier molecular orbitals. These parameters were used to understand the relationship of optical properties with the molecular structures. We found that the annealing process causes formation of nanostructures and the value of the third order optical susceptibility makes these materials interesting for future nonlinear optical applications.
\end{abstract}

\section{Introduction}

Phthalocyanine is an organic compound that forms stable complexes with several metals that have long been known as blue or green dyes and pigments. The metallophthalocyanine chlorides (MClPc, $\mathrm{M}=\mathrm{Al}$ and $\mathrm{Ga}$ ) are not only blue pigments, but also nontoxic p-type organic semiconductors with good thermal and chemical stability. These materials are extremely attractive for use in optical and electronic devices. The performances of these devices are strongly dependent on the nature of the material and its processing, design, and deposition technique. The factors including film fabrication technique, morphology of the film and the temperature of the annealing process are very important for the efficiency of optical or electronic devices.

Third order nonlinearities are usually described by the third order susceptibility $\chi^{(3)}\left(-\omega_{4} ; \omega_{3}, \omega_{2}, \omega_{1}\right)$, where the frequencies fulfill the following equation: $\omega_{4}=\omega_{3}+\omega_{2}+\omega_{1}$ and are of special interest since they provide a mechanism for all optical switching, i.e. control

\footnotetext{
* Corresponding author.

E-mail address: azawa@fizyka.umk.pl (A. Zawadzka).
}

of light by light. In the case where all frequencies have the same value $(\omega)$, the third order susceptibility $\chi^{(3)}(-\omega ; \omega,-\omega, \omega)$ describes effects that can also be conveniently viewed as light-induced changes in the refractive index of the nonlinear medium. The typically weak Van der Waals interactions in organic systems permit their optical characteristics, to a large extent, to be traceable to the properties of the constituent molecules. It has been well recognized in the field of third order optical nonlinearities that the third order hyperpolarizability $\gamma$, the microscopic analogy of $\chi^{(3)}$, attains high values for those molecules that possess extensive $\pi$ electron conjugation [1]. Macrocyclic molecules like phthalocyanines provide one example of a $\pi$-conjugated system that is virtually two dimensional, and therefore are interesting samples for studying optical nonlinearities. The properties of phthalocyanines are to some extent dependent on the presence and identity of the central metal atom. The role of the central metal atom has been investigated in some experiments [2-6]. A clear enhancement of the cubic hyperpolarizability has been observed for phthalocyanines containing cobalt, and the result has been justified on the basis of its uncompleted $d$-shell character $[2,3]$. More systematic data on the spectral dependence of the nonlinear response are needed in order to properly take into account the effect of 
frequency dispersion factors. Third Harmonic Generation (THG) experiments of spin-coated thin films of octaalkoxyphthalocyanines [7] and naphthalocyanines [8] also throw some light on the effects of metal substitution.

The main experimental techniques that have been used for third order nonlinear optical (NLO) processes of phthalocyanines are: THG [9], degenerate four-wave mixing (DFWM) [10] and Z-scan [11]. The primary emphasis of this work has been to measure $\chi^{(3)}$ for THG processes in $\mathrm{AlPcCl}$ and $\mathrm{GaPcCl}$ (Fig. 1). The main aim of this paper has been focused on a determination of the microscopic third order NLO behavior of the title phthalocyanine chlorides by the theoretical approaches and experimental investigation. So, we present here an ab-initio study utilizing time-dependent Hartree-Fock (TDHF) procedure on dispersion-free and frequencydependent dipole polarizabilities, second hyperpolarizabilities as well as THG measurements. The linear optical characterizations and $\chi^{(3)}$ values determined by UV-Vis and THG spectroscopies, respectively, for $\mathrm{AlPcCl}$ and $\mathrm{GaPcCl}$ have been compared with quantum mechanical calculations. The highest occupied molecular orbitals (HOMOs), the lowest unoccupied molecular orbitals (LUMOs) and the HOMO-LUMO band gaps have been also evaluated by density functional theory DFT calculations.

\section{Experimental methods}

\subsection{The film preparation}

The AlClPc and GaClPc thin films were fabricated by a Physical Vapor Deposition (PVD) technique using typical home-made equipment [12-16]. The thin films were successfully deposited on a quartz substrate. The process of fabrication was carried out under pressure about $3^{*} 10^{-3} \mathrm{~Pa}$. The powders of AlClPc and GaClPc $(97 \%$ Sigma-Aldrich Co.) were loaded into a quartz effusion cell with a nozzle diameter of $10 \mathrm{~mm}$. The source material was thermally evaporated from quartz crucible surrounded and heated by tungsten resistance coil. The temperature of the evaporation source was manually controlled (K-type thermocouple). The temperatures of the source for metallophthalocyanine chlorides were kept at $270{ }^{\circ} \mathrm{C}$ during whole evaporation process. The deposition rate was in the range $0.1-0.2 \mathrm{~nm} / \mathrm{s}$ and depended on the source material, its temperature and distance between the source material and the substrate. The quartz plates were located on the holder about $10 \mathrm{~cm}$ above the evaporation source. The thickness of fabricated AlClPc and $\mathrm{GaClPc}$ thin films was about $100 \mathrm{~nm}$. Substrates were held at room temperature during the deposition process. Selected samples were immediately subjected to an annealing process to assure different orientation of the molecules on the substrate, different nanostructures assembling on the surface and related linear (transmittance) and third order nonlinear optical properties.

\subsection{Characterization of nanostructural thin films}

Third Harmonic Generation (THG) measurements were carried out using the rotational Maker fringe technique [17] in the transmission scheme $[3,18]$. A fused silica glass plate was used as a reference material for THG measurements. A Q-switched modelocked Nd:YAG laser working at $1064 \mathrm{~nm}$ with $16 \mathrm{ps}$ pulse duration, $1.6 \mathrm{~mJ}$ power per pulse and the repetition frequency of $10 \mathrm{~Hz}$ was used as a fundamental laser beam. The fundamental beam was focused on the sample using a lens, whose focal distance was about $250 \mathrm{~mm}$. A diameter of the beam was equal to $0.4 \mathrm{~mm}$ at the film, which allowed achieving the power density equal to $5 \mathrm{GW} / \mathrm{cm}^{2}$. The intensity at the entrance to the sample was described by Gaussian distribution in space and time. A rotation stage with the mounted sample allowed the variation of the incidence angle, with a resolution of $0.5^{\circ}$. After passing the sample, the transmitting filter was used to cut the pump laser beam before the photomultiplier. Detector saturation was prevented using linear neutral density filters, whose transmittance value was taken into account during data fitting. The third harmonic signals were detected by the photomultiplier (HAMAMATSU R1828-01), integrated by a box-car average system and processed by a computer. A fast photodiode $(\mathrm{Ph} 2 \mathrm{~A})$ was used to monitor the input energy. Finally, the so-called Maker fringes were generated by rotating the sample through the range of $\pm 70^{\circ}$ to the fundamental laser beam and recorded.

The linear optical properties - the transmission spectra were measured at normal incidence in the spectral range 190-1100 nm using a double - beam spectrophotometer (Perkin Elmer Lambda 2 UV/VIS/NIR). In order to determine structural properties of the films, X-Ray Diffraction (XRD) and Atomic Force Microscopy measurements were taken. The AFM imaging was performed in the contact mode, with an Agilent 5500 instrument equipped with an MSNL-D Bruker cantilever. The process of formation of the crystalline phase and the type of the self-assembling nanonstructures
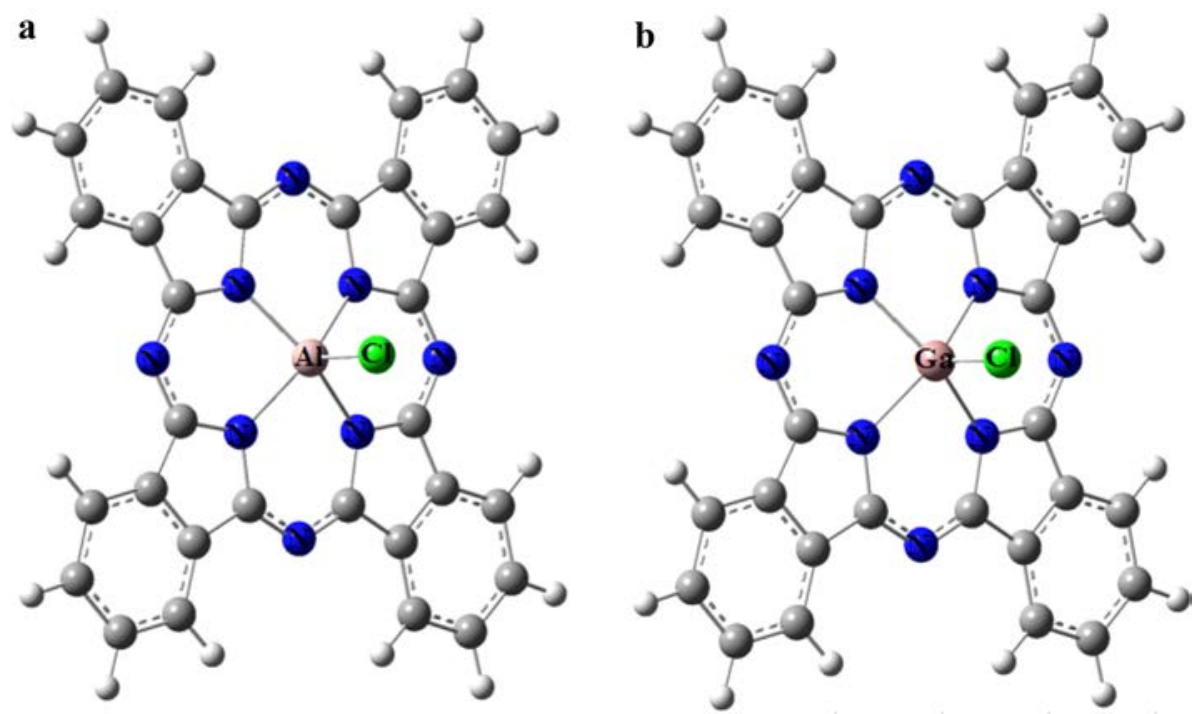

Fig. 1. Chemical structures of $\operatorname{AlClPc}(a)$ and GaClPc (b). 
for thermally treated samples were carried out by the X-Ray diffractometer using $\mathrm{CuK} \alpha(\lambda=0.1542 \mathrm{~nm})$ radiation.

\section{Theory of third harmonic generation process}

\subsection{Time-dependent Hartree-Fock (TDHF) method}

The theoretical computations involve the determination of dispersion-free and frequency-dependent dipole polarizability and third order hyperpolarizability tensor components. The molecule geometries of AlClPc and GaClPc have been initially optimized. The geometry optimizations have been followed by the calculations of static and dynamic dipole polarizabilities and second hyperpolarizabilities. The $6-311+\mathrm{G}(d, p)$ polarized and diffused basis set was found adequate for obtaining reliable hyperpolarizability values. Small molecules require quite extended basis sets [19] because the intra-atomic polarization is important and its accurate calculation needs a more precise choice of basis set, especially when it has diffuse functions which would be almost similar to describing an isolated atom. On the other hand, inter-atomic valence polarization is not in any case be required [20], it essentially depends on the size and geometric orientation of the molecule. However, when exploring the accurate determination of dipole hyperpolarizabilities both types of valence polarization require a careful choice of basis sets as reported by Maroulis [21], who utilized a systematic sequence of carefully optimized basis sets to calculate the static dipole hyperpolarizability of trans-butadiene. He stated that due to the large size of the molecule, the introduction of electron correlation effects required a stringent study of the basis sets, since calculations with small or insufficiently polarized basis sets might lead to unrealistic values. One expects the basis set $6-311+G(d, p)$ to yield molecular property values of near-Hartree-Fock quality. It has been also employed in the dipole polarizability and the third hyperpolarizability computations.

Knowledge of the frequency-dependent hyperpolarizabilities is required in order to make a direct comparison with experiment since all experiments involve at least one time-dependent field. This involves solution of the time-dependent Schrödinger equation. Thus, frequency-dependent hyperpolarizabilities are determined from analytic derivative calculations or using the sum-over-states formulation. Frequency-dependent hyperpolarizabilities have been frequently implemented at the self-consistent field level of theory (known as TDHF) [22]. With increasing molecular size the electronic hyperpolarizability becomes larger and its frequency dependence often becomes stronger [22]. $\alpha(0 ; 0)$ and $\gamma(0 ; 0,0,0)$ at $\omega=0, \alpha(-\omega ; \omega)$ and $\gamma(-3 \omega ; \omega, \omega, \omega)$ at $\omega=0.04282$ atomic units (a.u.) (i.e. at $\lambda=1064 \mathrm{~nm}$ wavelength) according to laser frequencies used in THG measurements have been carried out using the TDHF method with $6-311+\mathrm{G}(d, p)$ basis set implemented in the GAMESS [23] program. $\alpha(0 ; 0)$ and $\gamma(0 ; 0,0,0)$ definitions above mentioned describe the static dipole polarizability and third order hyperpolarizability, respectively. The frequency-dependent $\gamma(-3 \omega ; \omega, \omega, \omega)$ computations at considered $\omega$ frequency were carried out by THG group of the TDHF procedure. This method appeared to be a good compromise between accuracy and calculation duration.

In this work, the averaged (isotropic) dipole polarizability $\alpha$ and third order hyperpolarizability $\gamma$ values have been calculated using the following expressions:

$\alpha=\left(\alpha_{\mathrm{xx}}+\alpha_{\mathrm{yy}}+\alpha_{\mathrm{zz}}\right) / 3$

$\gamma=(1 / 5) \cdot\left[\gamma_{\mathrm{xxxx}}+\gamma_{\mathrm{yyyy}}+\gamma_{\mathrm{zzzz}}+2\left(\gamma_{\mathrm{xxyy}}+\gamma_{\mathrm{xxzz}}+\gamma_{\mathrm{yyzz}}\right)\right]$

We have also computed the orientationally averaged (isotropic) values of third order susceptibility $\chi^{(3)}(-\omega ; \omega,-\omega, \omega)$ which represent the nonlinear interaction of third-order. $\chi^{(3)}(-\omega ; \omega,-\omega, \omega)$ at $\omega=0.04282$ a.u. calculations were carried out by optical Kerr effect (OKE) group of the TDHF method with 6-311 + G $(d, p)$ basis set implemented in the GAMESS [24] program. To calculate all dipole polarizabilities, third order hyperpolarizabilities and susceptibilities, the origins of Cartesian coordinate systems $(x, y, z)=(0,0,0)$ have been chosen at the centres of masses of AlClPc and GaClPc.

Besides, the transition wavelengths $\left(\lambda_{\max }\right)$ of the lowest lying electronic transitions for AlClPc and GaClPc have been determined by CIDRT group (configuration interaction with all doubly occupied molecular orbitals from Hartree-Fock reference determinant) of the TDHF method using 6-31G basis set implemented in the GAMESS [24] program.

All OPA wavelengths, static $\alpha$ and $\gamma$, dynamic $\alpha, \gamma, \chi^{(3)}$ calculations have been performed on a PC with an Intel (R) core (TM) I72630QM operator, 5.8 GB RAM memory and $2 \mathrm{GHz}$ frequency using Linux PC GAMESS version running under Linux Fedora release 11 (Leonidas) environment.

To understand the relationship of NLO properties with the molecular structure; HOMOs, LUMOs and HOMO-LUMO gaps have been also generated by GAUSSIANO3W [25] program at DFT/B3LYP level with 6-31G basis set. The HOMO-LUMO band gap $\left(E_{\mathrm{g}}\right)$ could be expressed as follows:

$E_{\mathrm{g}}=E_{\mathrm{LUMO}}-E_{\mathrm{HOMO}}$

\subsection{Comparative model}

A few theoretical models, using various approximations, have been described in order to determine the value of the electronic contribution of the third order nonlinear optical susceptibility $\chi_{\text {elec }}^{<3>}$ from the shape of the experimental curves of Maker fringes $[17,26]$ obtained by the THG technique. We selected and used two of these models: the comparative model and the Reintjes model, for estimation of our experimental results.

The comparative model [27] (also known as the Lee model) compares directly the maximum of light intensities amplitudes for third harmonic of nonlinear medium with those of the reference material used for e.g. the experimental setup calibration. The value of the electric part of the third order nonlinear susceptibility $\chi_{\text {elec }}^{<3>}$ is derived by comparing third harmonic peak intensities of the investigated sample and reference material (fused silica glass for this experiment).

The comparative model allowed estimating the magnitude of the third order nonlinear susceptibility $\chi_{\text {elec }}^{<3>}$. The refractive indices and third order susceptibility were considered real. The $\chi_{\text {elec }}^{<3>}$ value of the investigated material was calculated using the following equation (4):

$\chi_{\text {elec }}^{3}=\chi_{\mathrm{S}}^{3}\left(\frac{2}{\pi}\right)\left(\frac{L_{\mathrm{CS}}}{d}\right) \sqrt{\left(I_{3 \omega \mathrm{S}} / I_{3 \omega}\right)}$

for the thin film whose thickness $d$ is much smaller than the coherence length $L_{\mathrm{CS}}$ of fused silica. The index $\mathrm{S}$ corresponds to the reference material, $\chi_{S}^{<3>}$ is the third order nonlinear optical susceptibility and $I_{3 \omega S}$ is the THG intensity of the reference measured under identical conditions to the sample. $I_{3 \omega}$ is the absorptioncorrected THG signal from the fringes of the sample. The value of $\chi_{S}^{<3>}$ for fused silica glass equals $3.11 \times 10^{-14}$ esu (at $\lambda_{\omega}=1064 \mathrm{~nm}$ ) and is reported in the literature [3,5].

\subsection{Theoretical model of Reintjes}

The model of Reintjtes was developed in 1984 [28]. In this model the wave equation is resolved for a homogenous and nonmagnetic nonlinear material, what explains the creation of Maker fringes. The shape of the THG spectrum changes with the angle of incidence 
$\theta_{\mathrm{i}}$. The Maker fringes become tighter when the angle increases because the length of the light inside the sample increases nonlinearly with the angle whereas the intensity of the fringes decreases due to the increase of reflection coefficient. The TH intensity is finally described by the following equation (5):

$I_{3 \omega}=\left[\frac{576 \pi^{6}}{n_{3 \omega} n_{\omega}^{3} \lambda_{\omega}^{2} c^{2}}\right]\left(\chi_{\text {elec }}^{3}\right)^{2} I_{\omega}^{2} L^{2}\left\{\frac{\sin \left(\pi L / 2 L_{C}\right)}{\pi L / 2 L_{C}}\right\}^{2}$

where: $L=\mathrm{d} / \cos \left[\operatorname{arc} \sin \left(\sin \theta_{\mathrm{i}} / n_{\omega}\right)\right]$ is the length of the lightpath in sample, $d$ is the thickness and $n_{\omega}$ is the refractive index of the investigated material, $\lambda_{\omega}$ is the fundamental wavelength and $L_{c}$ the distance along which bound and free waves gain a phase difference equal to $\pi$. The $L_{c}$ is described by the following relation: $6 \pi\left(n_{3 \omega}-n_{\omega}\right) / \lambda_{\omega}=\pi / L_{C}$.

\section{Results and discussion}

\subsection{X-Ray diffraction (XRD) study of AlClPc and GaClPc thin films}

Fig. 2a and b presents measurements of XRD ( $2 \theta$ scan $)$ of studied AlClPc and GaClPc thin films deposited on the substrate kept at room temperature during the PVD process and subsequently annealed at the temperature equal to $250^{\circ} \mathrm{C}$. The $2 \theta$ scan process is
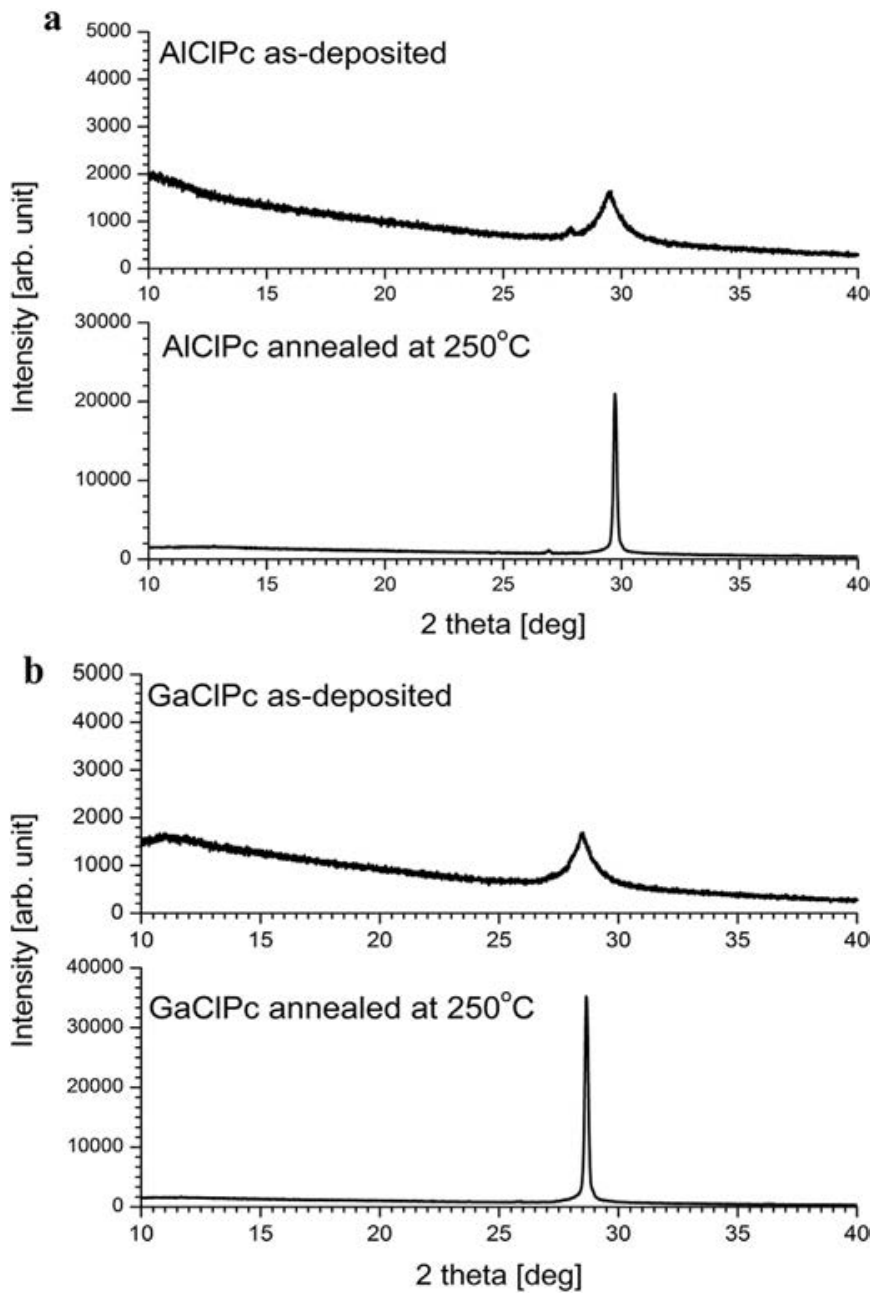

Fig. 2. XRD spectra of MClPc (M = Al, Ga) thin film: (a) as-deposited AlClPc sample and annealed at temperature of $250{ }^{\circ} \mathrm{C}$, (b) as-deposited GaClPc sample and annealed at temperature of $250^{\circ} \mathrm{C}$. sensitive to both reflections from parallel planes to the sample surface and others not fulfilling this condition. In the case of this experiment, all measurements were carried out by diffractometer using $\mathrm{CuK}_{\alpha}(\lambda=0.1542 \mathrm{~nm})$ radiation.

One strong peak was observed in most cases of the measured XRD spectra. The spectra show that the films have a well-defined orientation of crystallization, especially after the annealing process. For the as-deposited GaClPc thin film there is only one small and relatively wide peak with the maximum at $2 \theta=28.517^{\circ}$. For the sample annealed at a temperature equal to $250{ }^{\circ} \mathrm{C}$ one peak is also observed, but the intensity of the peak is about 25 higher. The position of the peak is slightly shifted toward larger values of the $2 \theta$ angle and the maximum of the peak is located at $2 \theta=28.665^{\circ}$. For the as-deposited AlClPc thin film there are two small and relatively wide peaks: one with the maximum at $2 \theta=29.520^{\circ}$ and second at $27.724^{\circ}$. For the sample annealed at a temperature equal to $250{ }^{\circ} \mathrm{C}$ two peaks are also observed, but the location and intensity of each one behaves differently as the temperature increases. The position of the first peak is slightly shifted toward larger values of the $2 \theta$ angle, the maximum of the peak is located at $2 \theta=29.723^{\circ}$ and the intensity of the peak is higher. The position of the second peak is much more shifted toward smaller values of the $2 \theta$ angle, the maximum of the peak is located at $2 \theta=26.886^{\circ}$ and the intensity of the peak is comparable. For all annealed films, the intensity of the peaks increases with increasing annealing temperature and the location of the peaks shifts mostly towards larger values of the $2 \theta$ angle. The growth of the intensity leads to the conclusion, that higher annealing temperature causes the higher level of the crystal structure arrangement. This phenomenon can be explained by various particle mobility as a function of the temperature. The particles have higher surface mobility in higher temperature, which provides the possibility of taking up defined positions on the surface. Application of the annealing process immediately after the deposition allows specifying the geometry of the particles assembly and therefore self-assembling organometallic nanostructures formation. Self-assembly is an equilibrium process where the assembled components are in equilibrium with the surrounding components. Self-assembly is driven by the minimization of Gibbs free energy. The minimization of Gibbs free energy is attained by the minimization of repulsive and the maximization of attractive molecular interactions. In addition, the lower free energy is usually a result of the weaker intermolecular force between self-assembled moieties. Hence, orientation of layers obtained in higher temperature is more arranged. For lower temperatures this orientation is more accidental and weaker particles' mobility counteracts crystallization of layer.

A half-width (FWHM) of all peaks decreased significantly with increasing temperature of the annealing process. The shape of the peaks also reflects the higher degree of crystallization, because of the greater size of nanostructures including sharper XRD peaks.

The grain size $\mathrm{L}$ of the self-assembled nanostructures was calculated using the Scherrer formula [29]:

$L=\frac{K \lambda}{b \cos (\theta)}$,

where $\lambda$ is the wave length of the $\mathrm{X}$-ray beam $(\lambda=0.1542 \mathrm{~nm}), b$ is the value of the full width at half maximum (FWHM), $\theta$ is the corresponding Bragg angle and $K$ is Scherrer constant. The value of $K$, in general, depends on the crystallite shape and it is assigned a value of 0.9 for phthalocyanine films [30]. The grain sizes corresponding to the as-deposited and annealed nanostructures are calculated and given in Table 1.

Inspection of Table 1 shows that as the annealing temperature increases, the FWHM decreases, providing that the crystallite size 
Table 1

Observed position of the most intense peak ( $2 \theta)$, FWHM (b), maximum intensity and grain size (L) for $\mathrm{MPcCl}$ nanostructures.

\begin{tabular}{lcccl}
\hline MCIPc sample & $2 \theta[\mathrm{deg}]$ & $\begin{array}{l}\text { FWHM }(\mathrm{b}) \times \\
10^{-3}[\mathrm{rad}]\end{array}$ & $\begin{array}{l}\text { Max. Intensity } \\
{[\mathrm{Cps}]}\end{array}$ & $\mathrm{L}[\mathrm{nm}]$ \\
\hline AlClPc as-deposited & 29.520 & 12.54 & 1626 & 21,474 \\
AlClPc annealed $250{ }^{\circ} \mathrm{C}$ & 29.723 & 2.41 & 21001 & 110,928 \\
GaClPc as-deposited & 28.517 & 15.41 & 1691 & 18.099 \\
GaClPc annealed $250^{\circ} \mathrm{C}$ & 28.665 & 2.45 & 35455 & 113.057 \\
\hline
\end{tabular}

increases from $21.474 \mathrm{~nm}$ for the as-deposited AlClPc sample to $110.928 \mathrm{~nm}$ for the sample annealed at $250^{\circ} \mathrm{C}$ and from $18.099 \mathrm{~nm}$ for the as-deposited GaClPc sample to $113.057 \mathrm{~nm}$ for the sample annealed at $250{ }^{\circ} \mathrm{C}$. The observed decrease in the FWHM and the increase in the crystallite size, due to annealing, are in good agreement with the results previously obtained for metallophthalocyanine chlorides [31]. The increase in the crystallinity is caused by the self-assembling nanostructures' formation and the phase change of the sample.

\subsection{Atomic force microscopy of AlClPc and GaClPc thin films}

In the case of metallophthalocyanine chloride nanostructures the knowledge of the surface morphology and the preferred orientation of the crystallites are essential for their prospective applications. Especially, the analysis of preferred molecule orientation and the tilt angle between the plane of molecule and the substrate play an important role and are related to their optical properties.

It is well known, that the arrangement of molecular orientations during the fabrication process strongly depends on growth conditions and the type of the substrate. Our investigations show also that the annealing process applied immediately after the deposition can significantly change the structural and optical properties. PVD process involves depositing a source material (powders of Aland GaClPc, 97\% Sigma-Aldrich Co) onto the substrate (quartz). During this process particles are typically randomly dispersed inside the evaporated plume and form randomly ordered nanostructures (see Fig. 3a and c) on the substrate's surface. The MCIPcs molecules are adsorbed on the substrate several seconds after the immersion of the substrate plate. Application of the annealing process immediately after the deposition allows specifying geometry of the particle assembly and therefore self-assembling organometallic nanostructure formation. The temperature equal to $250{ }^{\circ} \mathrm{C}$ and the time period equal to $24 \mathrm{~h}$ allowed achieving the new arrangement of molecules, finding the minimum Gibbs free energy of the system and optimizing all complicated interactions between the adsorbed MClPcs molecules. Using the annealing process, we obtained nanostructures with a specific arrangement parallel to each other and almost parallel to the substrate, as illustrated in Fig. $3 \mathrm{~b}$ and $\mathrm{d}$. The nanostructures like nanorods are especially interesting and may have applications in nonlinear optical application as well as thermal sensing production. This irreversible thermally-induced self-assembly process is versatile, as a single assembly system can pattern multiple geometries by simply controlling the external thermal condition. The process is also scalable and cost-effective, presenting itself as a promising manufacturing tool of nanostructures. The ability to assemble nanostructures with well-defined configuration in space is crucial to the development of electronic devices that are small but can contain plenty of information. The spatial arrangements of these self-assembled nanorods can be potentially used to build increasingly complex structures leading to a wide variety of materials that can be used for different purposes.

\subsection{UV-Vis spectroscopy}

The transmittance $\mathrm{T}(\lambda)$ of the nanostructures was measured at normal incidence in the spectral range $190-1100 \mathrm{~nm}$. The spectral distribution of transmittance was measured for as-deposited and annealed samples. Fig. $4 \mathrm{a}$ and b presents measurements of AlClPc and $\mathrm{GaClPc}$ transmittance for samples only deposited without annealing process as well as samples deposited and next annealed at $150{ }^{\circ} \mathrm{C}$ for $24 \mathrm{~h}$ and samples deposited and next annealed at $250{ }^{\circ} \mathrm{C}$ for $24 \mathrm{~h}$. Review of Fig. 4 shows that transmittance intensity of the formed nanostructures can change the value after the annealing process with a shift of all minima. Annealing at $150{ }^{\circ} \mathrm{C}$ doesn't change its value for AlClPc and decreases the transmittance for GaClPc. Annealing at $250{ }^{\circ} \mathrm{C}$ decreases the transmittance for both tested MClPcs compounds in comparison with transmittance of samples before application of the process. Annealing at both temperatures shifts the peak position of all bands towards high wavelength side of spectra.

The electronic level structure of phthalocyanines has been investigated by several authors [32-34]. For metal-free phthalocyanines, the $\mathrm{Q}$ band is typically split into two main components while metal-containing phthalocyanines exhibit only a single $Q$ band in their visible spectra. The exact position of these bands depends on the particular structure, metal complexation and peripheral substituents [33,34]. Metal incorporation induces a decrease in the extinction coefficient for the $\mathrm{Q}$ (and B) band. For peripherally substituted metallic phthalocyanines (e.g. push-pull compounds), the degenerate $\mathrm{Q}$ bands show some splitting due to the reduction in symmetry.

In all investigated samples regardless of the annealing process, four absorption bands take notations $\mathrm{Q}, \mathrm{B}, \mathrm{N}$ and $\mathrm{C}$ were observed. The Q-band exists in the visible region of spectra while others (B, N and $C$ ) exist in the UV region of spectra. It is also noted that the bands $\mathrm{B}, \mathrm{N}$ and $\mathrm{C}$ appear with comparative intensities to the $\mathrm{Q}$ bands.

Analysis of the spectra (Fig.4) clearly shows the appearance of Q-band splitting into two bands (around 640-760 nm) for both MClPcs, regardless of whether the sample was subjected to an annealing process or not. However, the intensity of the minima and their position as a function of wavelength changed substantially depending on the temperature of the annealing process. There is a shift towards larger wavelengths in the case of all MClPcs, but with one exception. The exception is aluminum phthalocyanine chloride annealing at temperature $250{ }^{\circ} \mathrm{C}$. In this case, the whole spectrum is shifted towards shorter wavelength. A thorough analysis of UV-VIS region shows that the B-band splitting also occurs for both investigated compounds (around $350-380 \mathrm{~nm}$ ).

It is well known that molecules of the metallophthalocyanine chlorides or similar compounds may exist mainly in two forms: monomer and dimer. In the dimer form, the close proximity of the two rings can lead to coupling between the transition moments for two identical molecules. This interaction, gives rise to two new levels of energy and the separation between them, is referred to the exciton splitting energy.

In order to describe this phenomenon, the so-called physical dimer should be considered. This term defines the relationship created by two identical molecules, which are so close to each other and form relative orientation, the same as in the crystal lattice. The relationship and interaction between the two molecules cannot be neglected. The Hamiltonian operator for the physical dimer is written as the sum of energy operators of both isolated molecules and the factor representing this intermolecular interaction [12]. Physical dimer ground state energy is expressed by the sum of the ground states energies and so-called Coulomb interaction energy. If 

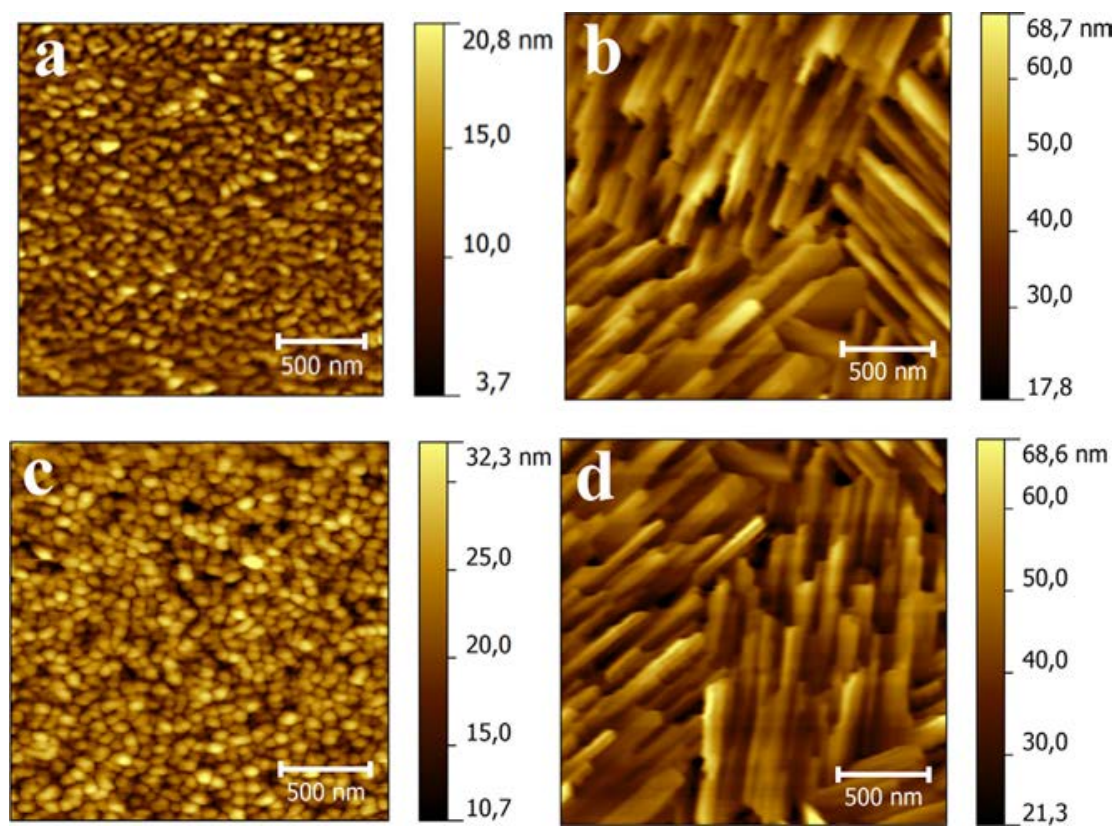

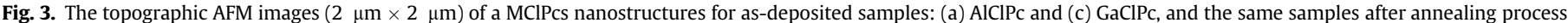
at temperature equal to $250{ }^{\circ} \mathrm{C}$ (b) AlCIPc and (d) GaClPc.

the molecular interaction is different from zero, the energy of electronic excitation isn't localized on one molecule, but begins to oscillate between the two dimer molecules and the probability of the molecule excitation is the same for each dimer molecule.
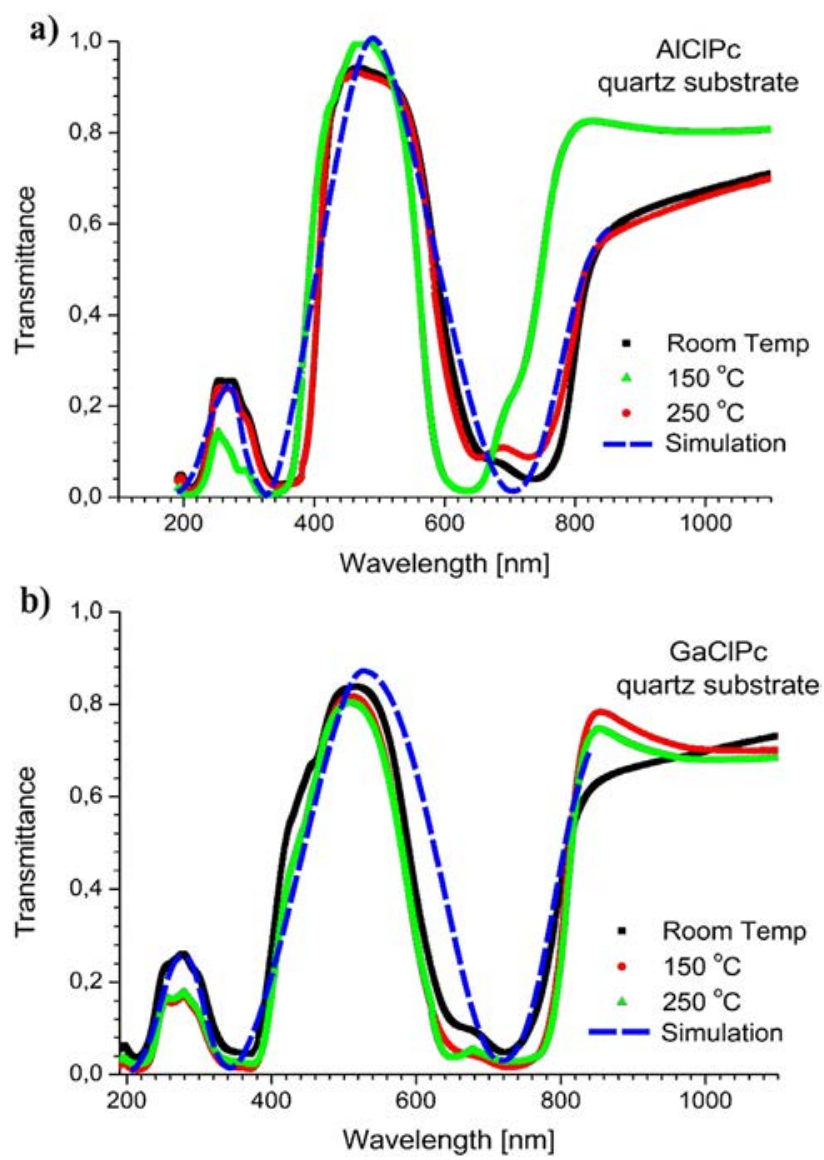

Fig. 4. Experimental transmittance spectra and theoretical simulations of MCIPcs: (a) AlClPc and (b) GaClPc.
In real crystals the molecules form a three-dimensional network. Moreover, most organic molecular crystals contain differently oriented molecules in the unit cell. The presence of more than one (interacting) equivalent molecular entity in the unit cell causes the splitting of bands in the electronic or vibrational spectra of crystals - so called Davydov splitting. The magnitude of the splitting depends on the intermolecular distance. If there are two molecules in the unit cell, two exciton bands will be present in the spectrum the band shift may occur in both directions, longer or shorter wavelengths. It depends on the mutual orientation of transition dipole moments of molecules forming the dimer. The numerical values of the Davydov Q-band's splitting for materials of interest here are presented in Table 2.

The two Davydov components give different polarization contributions thus different optical transitions probability. The differences in polarization result from the symmetry of the molecules and the formed nanostructures. The strength of the resonance interaction, which is responsible for the Davydov splitting, can be calculated for singlet state, and when the transition is allowed in the free molecule, as the Coulomb interaction of transition dipole moment of the excited state with the neighboring molecules in their ground states.

The broad absorption peak between the B and Q bands could be used for frequency doubling into the green spectral region. It is commonly accepted that those bands are primarily derived from transitions between the highest occupied molecular orbitals, HOMO and HOMO-1, and the two lowest unoccupied molecular orbitals, LUMO and LUMO + 1. In this paper, the vertical transition energies from the ground state to each excited state for AlClPc and $\mathrm{GaClPc}$ have been computed, giving optical absorption, i.e. the UV-Vis spectrum. The calculated wavelengths $\left(\lambda_{\max }\right)$ for the maximum of the optical absorption are shown in Table 3. The computed $\left(\lambda_{\max }\right)$ values are also in good agreement with the experimental results recorded for the MCIPcs nanostructures. AlClPc and GaClPc exhibit electronic transitions in the visible (two $\mathrm{Q}$ bands at $470-805 \mathrm{~nm}$ for AlClPc and at $520-848 \mathrm{~nm}$ for GaClPc) and in the UV (one B or Soret band at around $270-300 \mathrm{~nm}$ for both phthalocyanines) regions as shown in Fig. 4 and Table 3. Both the $\mathrm{Q}$ and $\mathrm{B}$ bands arise from transitions to $E_{\mathrm{g}}$ states. The $\mathrm{Q}$ bands are 
Table 2

The energy values of Q-band's and Davydov splitting for MClPcs.

\begin{tabular}{lllll}
\hline Sample & $\begin{array}{l}\text { Annea-ling } \\
\text { temp. }\left[{ }^{\circ} \mathrm{C}\right]\end{array}$ & \multicolumn{2}{l}{$\mathrm{Q}-$ band energies } & $\begin{array}{l}\text { Davydov } \\
\text { splitting }[\mathrm{nm}]\end{array}$ \\
\cline { 2 - 4 } & & $E_{1}[\mathrm{~nm}]$ & $E_{2}[\mathrm{~nm}]$ & \\
\hline AlClPc & - & 664.71 & 734.23 & 69,52 \\
& 150 & 661.62 & 732.49 & 70,87 \\
& 250 & 633.15 & 711.96 & 78,81 \\
GaClPc & - & 653.70 & 721.45 & 67,75 \\
& 150 & 655.23 & 727.84 & 72,61 \\
& 250 & 664.72 & 737.33 & 72,61 \\
\hline
\end{tabular}

strongly localized on the phthalocyanine rings. In many cases, it has been found that the $\mathrm{Q}$ band is very sensitive to the environment of the molecule, and is also more sensitive to changes in the number and the orientation of nearest neighbor phthalocyanines in the solid state [35]. For main group elements, such as the Al and Ga [36], the filled $4 d$ orbitals are below the filled $\pi$ ring levels and the unfilled $4 s$ orbitals are above the $\pi^{*}$ orbitals. Thus, the central metal does not directly participate in the electronic transition. The most widely used method for calculating properties of electronically excited states is configuration interaction (CI). One starts from the Hartree-Fock wave function of the corresponding electronic ground state and performs a $\mathrm{CI}$ calculation. In this way, one can obtain a reasonably accurate description of the optical spectrum of the system. The calculated vertical transition energies in Table 3 are systematically only slightly larger than the experimental values. The overestimated excitation energy may originate from the truncated $\mathrm{CI}$ method in this work that does not correlate excited states as well as the ground states.

We have examined the molecular HOMO and LUMO energies of AlClPc and GaClPc. The HOMO and LUMO energies and HOMO-LUMO energy gaps of the studied molecules determined by DFT method at B3LYP/6-31G level are listed in Table 4. The DFT has become a popular alternative to traditional ab-initio electronic structure methods in calculating molecular properties. The DFT theory was chosen to compute the energies of frontier molecular orbitals of our systems due to its reliability already discussed [37]. However, the calculations performed by this method are always with underestimated HOMO-LUMO gap. The frontier molecular orbitals are shown in Figs. 5 and 6. In these figures, HOMO- 1 refers to the second highest occupied molecular orbital. It is known that the molecular electronic spectrum is usually caused by the electron transition from the HOMO to the LUMO. The second and third order NLO responses can be dictated by charge transfer excitations involving the HOMO and LUMO orbitals in such a way that larger values of second and third order hyperpolarizabilities should correspond to lower HOMO-LUMO gaps. Therefore, it could be expected that both investigated molecules with rather low HOMO-LUMO gaps might have third order optical nonlinearity with non-zero responses. The HOMO in GaClPc is localized on both phthalocyanine ring and also aromatic benzene rings, whereas the reduction of localization at HOMO-1 occurs on the phthalocyanine

Table 3

Calculated and experimentally observed maximum wavelengths of the absorption for AlCIPc and GaClPc.

\begin{tabular}{lll}
\hline Compound & $\lambda_{\max }(\mathrm{nm})$ & $\lambda_{\exp }(\mathrm{nm})$ \\
\hline AlClPc & 286.30 & 270 \\
& 493.11 & 470 \\
GaClPc & 804.00 & 780 \\
& 299.26 & 280 \\
& 558.39 & 520 \\
& 847.45 & 830 \\
\hline
\end{tabular}

Table 4

Calculated HOMO-LUMO energy (a.u.) and HOMO-LUMO band gap $\left(E_{\mathrm{g}}\right)$ values for AlClPc and GaClPc.

\begin{tabular}{lrr}
\hline Compound & AlClPc & GaClPc \\
\hline HOMO & -0.19634 & -0.19640 \\
LUMO & -0.11723 & -0.11666 \\
$E_{g}[$ HOMO - LUMO] & 0.07911 & 0.07974 \\
HOMO -1 & -0.25359 & -0.25710 \\
LUMO + 1 & -0.11723 & -0.11666 \\
$E_{g}[($ HOMO-1) - (LUMO +1$)]$ & 0.13636 & 0.14044 \\
\hline
\end{tabular}

ring (Fig. 6). The localized HOMO value on both phthalocyanine ring and aromatic benzene ring and HOMO-1 value on symmetrically two aromatic benzene rings in AlClPc have been obtained to be lower than the values in GaClPc (Figs. 5 and 6). The LUMO and LUMO + 1 have been determined as equal values in AlClPc $(-0.11723$ a.u.) and in $\mathrm{GaClPc}(-0.11666$ a.u.). GaClPc has smaller LUMO (and LUMO + 1) localized on the phthalocyanine ring and especially symmetrically two aromatic benzene rings than that of AlClPc, leading to higher values of band gaps (0.07974 a.u. and 0.14044 a.u.) (see Table 4).

\subsection{THG measurements and computational results}

There is a larger variety of third order processes and susceptibilities [25] in comparison to second order ones. Parametric processes include THG, four-wave mixing, intensity-dependent refractive index and Kerr electro-optic effects. Each process can be associated with one or more various mechanisms whose relative importance depends on experimental conditions. Among the main techniques [38] that have been used for phthalocyanines and related compounds, THG measures $\chi^{(3)}(-3 \omega ; \omega, \omega, \omega)$ and exclusively
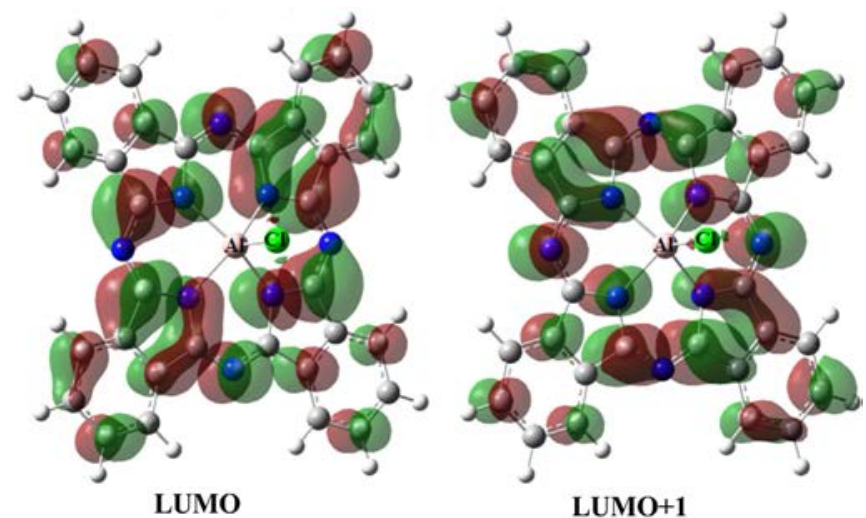

LUMO+1
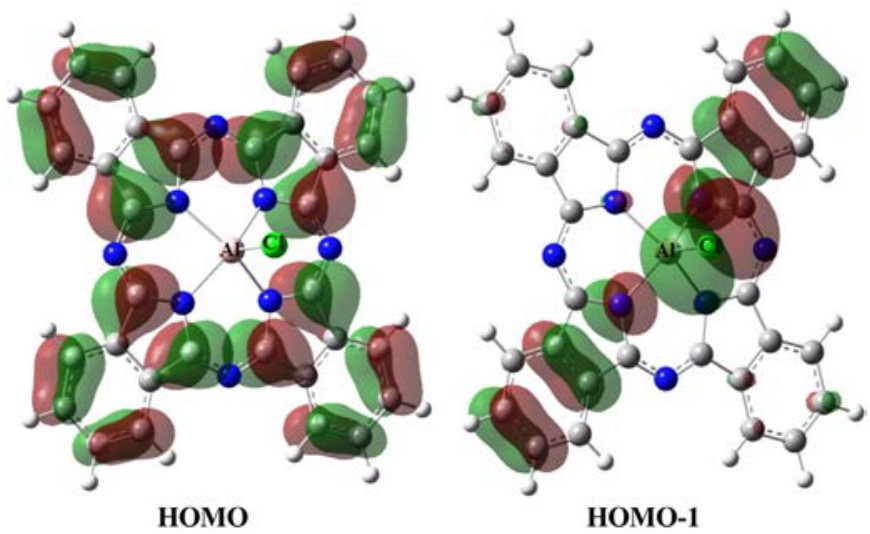

Fig. 5. The frontier and second frontier molecular orbitals of AICIPC. 

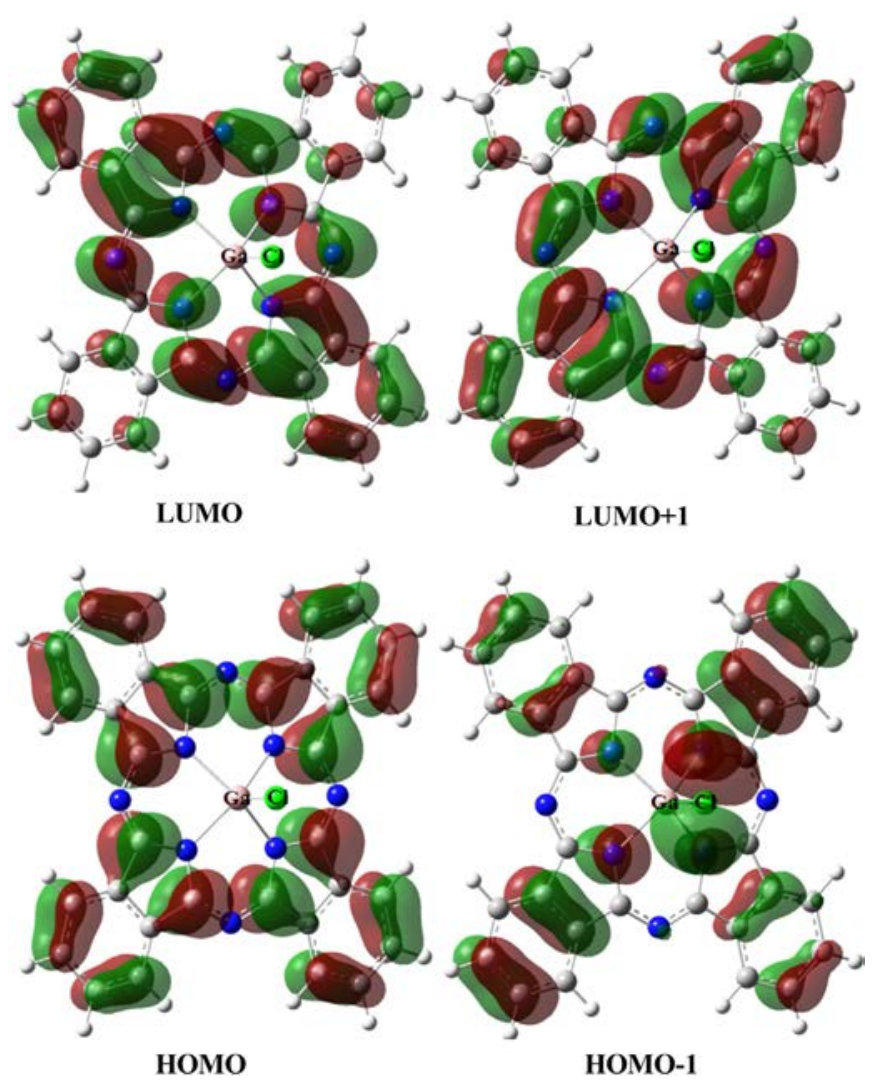

Fig. 6. The frontier and second frontier molecular orbitals of GaClPc.

involves electronic mechanisms. At variance with THG, Degenerate Four-Wave Mixing (DFWM) and Z-scan are sensitive to nonelectronic mechanisms. Therefore, for third order effects, one must be especially careful when comparing data obtained by different techniques. Also, even using the same technique, differences in the wavelength and pulse length of the fundamental beam may give rise to different results due to the different dispersion laws and time scales (dynamics) associated with each particular mechanism.

In spite of the rapid development of highly advanced experimental techniques on NLO, the theoretical understanding of the NLO properties show that the responses of the molecule to the external application of an electric field are also of much importance. One could determine the hyperpolarizability tensors of molecules using a suitable computational approach. At the molecular level, the NLO properties are determined by their dynamic hyperpolarizabilities. We present here an ab-initio calculation on the NLO properties of AlCIPc and GaClPc using the TDHF method. In this work, in addition to the static dipole polarizabilities $\alpha(0 ; 0)$ and third order hyperpolarizabilities $\gamma(0 ; 0,0,0)$, the following processes for dynamic hyperpolarizabilities have been considered: dispersions of dipole polarizabilities $\alpha(-\omega ; \omega)$ and third order hyperpolarizabilities $\chi^{(3)}(-3 \omega ; \omega, \omega, \omega)$. Some significant calculated magnitudes of the static and dynamic dipole polarizabilities and

Table 5

Some selected components of the static $\alpha(0 ; 0)$ and $\alpha(0 ; 0)\left(\times 10^{-24}\right.$ esu) values for AlClPc and GaClPc.

\begin{tabular}{lllll}
\hline Compound & $\alpha_{\mathrm{xx}}$ & $\alpha_{\mathrm{yy}}$ & $\alpha_{\mathrm{zz}}$ & $\alpha$ \\
\hline AlClPc & 72.875 & 67.539 & 13.637 & 51.350 \\
GaClPc & 72.935 & 67.653 & 13.754 & 51.447 \\
\hline
\end{tabular}

Table 6

Some selected components of the frequency-dependent $\alpha(-\omega ; \omega)$ and $\alpha(-\omega ; \omega)\left(\times 10^{-24}\right.$ esu $)$ values at $\omega=0.04282$ a.u. $(\lambda=1064 \mathrm{~nm})$ for AlClPc and GaClPc.

\begin{tabular}{lllll}
\hline Compound & $\alpha_{\mathrm{xx}}$ & $\alpha_{\mathrm{yy}}$ & $\alpha_{\mathrm{zz}}$ & $\alpha$ \\
\hline AlClPc & 77.173 & 69.605 & 13.828 & 53.535 \\
GaClPc & 77.225 & 69.719 & 13.946 & 53.630 \\
\hline
\end{tabular}

third order hyperpolarizabilities are shown in Tables 5-8, respectively.

The computed values of dynamic $\alpha$ and $\gamma$ for AlClPc and GaClPc have very little enhancement in the order AlClPc < GaClPc (see Tables 6 and 8). Diaz-Garcia et al. [39] measured the cubic hyperpolarizability value by means of THG of a phthalocyanine containing cobalt to be $\gamma=44800\left(\times 10^{-37} \mathrm{esu}\right)$ at $\lambda=1340 \mathrm{~nm}$. This result is almost 30 times higher than the dynamic $\gamma$ values of AlCIPc and $\mathrm{GaClPc}$ (Table 8). In this sense, incorporation of cobalt seems to more strongly enhance the third order optical nonlinearity comparing with $\mathrm{Al}$ and $\mathrm{Ga}$ metals. Sastre et al. [40] reported the absolute value of second hyperpolarizability of $(t-\mathrm{Bu})_{4} \mathrm{PcH}_{2}$ (Pc: phthalocyanine) using THG experiment at $\lambda=1340 \mathrm{~nm}(\gamma=14000$ $\left(\times 10^{-37}\right.$ esu)). Our computations on dynamic $\gamma$ for AlClPc and $\mathrm{GaClPc}$ are about factor of 9 lower than the experimental finding obtained by Sastre et al. [40]. We give the averaged values of third order susceptibilities obtained theoretically of AlClPc and GaClPc (see Table 9). Our calculation results on $\chi^{(3)}$ are in good agreement with the experimental data, indicating an enhancement in the order AlClPc $<$ GaClPc. It is also seen from Table 8 that the theoretical data on dynamic third order hyperpolarizabilities $\gamma$ have the same enhancement with very small variation in the order AlClPc $<$ GaClPc as the values of third order susceptibilities (Table 9). The third order susceptibilities of naphthalocyanines $\mathrm{MnCl}[\mathrm{Pc}(\mathrm{OPh}) 8]$ and $\mathrm{MnCl}[\mathrm{Pc}(\mathrm{OC} 8 \mathrm{H} 17) 8]$ in toluene solution were studied using Z-scan technique by Sun et al. [41] $\left(\chi^{(3)}=1.44(\times\right.$ $\left.10^{-11} \mathrm{esu}\right)$ and $2.27\left(\times 10^{-11} \mathrm{esu}\right)$ at $\lambda=532 \mathrm{~nm}$, respectively). The third order susceptibilities of naphthalocyanines with metal substitutions, $\left(\mathrm{SC}_{10} \mathrm{H}_{21}\right)_{4} \mathrm{NcSi}\left[\mathrm{OSi}\left(\mathrm{C}_{2} \mathrm{H}_{5}\right)_{3}\right]_{2}$ and $\left(\mathrm{CO}_{2} \mathrm{C}_{5} \mathrm{H}_{11}\right)_{4} \mathrm{NcGe}$ $\left[\mathrm{OSi}\left(\mathrm{C}_{4} \mathrm{H}_{9}\right)_{3}\right]_{2}$ (Nc: naphthalocyanine), were evaluated using THG experiments by Nalwa et al. [42] $\left(\chi^{(3)}=5.60\left(\times 10^{-11} \mathrm{esu}\right)\right.$ and 1.86 $\left(\times 10^{-11} \mathrm{esu}\right)$ at $\lambda=1500 \mathrm{~nm}$, respectively). $(t-\mathrm{Bu})_{4} \mathrm{PcGe}(\mathrm{OH})_{2}$ exhibited the $\chi^{(3)}=1.05\left(\times 10^{-11}\right.$ esu $)$ value at $\lambda=1500 \mathrm{~nm}$ by means of THG measurements [42].

In this work, the third order susceptibilities of AlClPc and GaClPc have been measured and calculated to be about $10^{-11}$ esu, quite consistent magnitude as compared to the cases of the other reported metallophthalocyanines $[8,41,42]$.

In order to obtain experimental information about the nonlinear optical properties AlClPc and GaClPc compounds were investigated by using THG technique. Nanostructures of MCIPcs deposited on a $1 \mathrm{~mm}$ thick quartz substrate were measured. THG signals were analyzed using the Maker fringes technique with fused silica as a reference material. Figs. 7 and 8 show the Maker fringes patterns of MClPc samples for S - vertical (Figs. 7a and 8a) and P - horizontal (Figs. $7 \mathrm{~b}$ and $8 \mathrm{~b}$ ) polarization of the laser radiation. It can be seen that the curve displays an oscillatory signal and intensities depend on the incidence angle. Good symmetry of THG signal for both as

Table 7

All static $\gamma(0 ; 0,0,0)$ components and $\gamma(0 ; 0,0,0)\left(\times 10^{-37}\right.$ esu $)$ values for AlClPc and GaClPc.

\begin{tabular}{llllllll}
\hline Compound & $\gamma_{\mathrm{xxxx}}$ & $\gamma_{\mathrm{yyyy}}$ & $\gamma_{\mathrm{zzzz}}$ & $\gamma_{\mathrm{xxyy}}$ & $\gamma_{\mathrm{xxzz}}$ & $\gamma_{\mathrm{yyzz}}$ & $\gamma$ \\
\hline AlClPc & 1752.937 & 1564.344 & 26.528 & 465.145 & 20.870 & 44.558 & 880.991 \\
GaClPc & 1743.656 & 1554.413 & 23.762 & 461.345 & 21.049 & 43.498 & 874.723 \\
\hline
\end{tabular}


Table 8

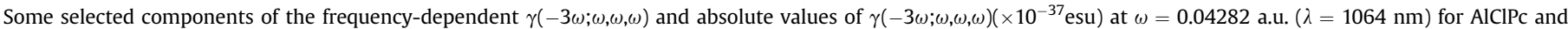
GaClPc.

\begin{tabular}{|c|c|c|c|c|c|c|c|}
\hline Compound & $\gamma_{\mathrm{xxxx}}$ & $\gamma_{\text {yyyy }}$ & $\gamma_{\mathrm{zzzz}}$ & $\gamma_{\text {xxyy }}$ & $\gamma_{x x z z}$ & $\gamma_{\text {yyzz }}$ & $\gamma$ \\
\hline AlClPc & 2290.479 & 232.802 & 62.173 & -11102.618 & -224.008 & 41.014 & 1435.402 \\
\hline $\mathrm{GaClPc}$ & 2228.329 & 229.667 & 51.274 & -11069.697 & -228.311 & 31.398 & 1454.386 \\
\hline
\end{tabular}

deposited and annealed MClPc samples were found, what proved the high homogeneity and good crystallinity. It was found that the intensity of the signals is slightly dependent on the annealing process temperature and almost independent on the laser polarization for all THG measurements.

From Figs. 7 and 8, one can see a non-monotonic and a symmetric angle dependence of the THG signal which reflects a substantial optical nonlinearity. This symmetry was characteristic and typical for both investigated metallophthalocyanine chlorides regardless of the annealing process. These experimental results and simply theoretical calculation based on described Reintjes model allow estimating the value of the real part of the third order nonlinear optical susceptibility $\chi_{\text {elec }}^{<3>}$. The theoretical model of Reintjes is the most adapted for explaining the experimental values of THG for these organometallic compounds, because it takes into account the majority of parameters which influence the value of $\chi_{\text {elec }}^{<3>}$ like the contribution of transmission factors on the interfaces of the nonlinear medium. In the case of the presented experiment, the results obtained with two models (Comparative and Reintjes) give similar values of the third order nonlinear optical susceptibility $\chi_{\text {elec }}^{<3>}$ for both as-deposited and annealed samples.

Nonlinear optical properties of MClPcs complexes depend mainly on their molecular composition such as an atomic number, position and nature of substituent metal atoms or molecular complexes. A size of the central metal and its position relative to the ring of metal-free phthalocyanine play the most important role. Metallophtalocyanine chlorides represent a large family of materials with centrosymmetric planar structure of 16 member rings with 18 delocalized $\pi$-electrons which leads to their special optical properties and extraordinary morphological and thermal stability over a larger temperature range compared to metallo- or unsubstituted phthalocyanines. The structure of the MCIPc differs from metal-free phthalocyanine and is similar to the MPc oxides. The Al

Table 9

The real part of the third order nonlinear optical susceptibility $\chi_{\text {elec }}^{<3>}$ measured values at $1064 \mathrm{~nm}$ and calculated averaged (isotropic) $\chi^{(3)}(-\omega ; \omega,-\omega, \omega)$ third order susceptibilities at $\omega=0.04282$ a.u. $(\lambda=1064 \mathrm{~nm})$ for AlClPc and GaClPc.

\begin{tabular}{lllr}
\hline Sample & Polarization & $\begin{array}{l}\text { Annealing process } \\
\text { temperature }\left[{ }^{\circ} \mathrm{C}\right]\end{array}$ & $\chi^{(3)}\left[10^{-11} \mathrm{esu}\right]$ \\
\hline AlCIPc & $\mathrm{P}$ & As deposited & $7.31 \pm 0.06^{\mathrm{a}}$ \\
& $\mathrm{S}$ & As deposited & $7.52 \pm 0.06^{\mathrm{a}}$ \\
& $\mathrm{P}$ & 150 & $8.31 \pm 0.06^{\mathrm{a}}$ \\
& $\mathrm{S}$ & 150 & $8.12 \pm 0.10^{\mathrm{a}}$ \\
& $\mathrm{P}$ & 250 & $7.10 \pm 0.15^{\mathrm{a}}$ \\
& $\mathrm{S}$ & 250 & $6.53 \pm 0.14^{\mathrm{a}}$ \\
& $\mathrm{P}, \mathrm{S}$ & & $7.96 \pm 0.09^{\mathrm{b}}$ \\
GaClPc & $\mathrm{P}, \mathrm{S}$ & As deposited & $9.358 \pm 0.1^{\mathrm{c}}$ \\
& $\mathrm{P}$ & As deposited & $9.40 \pm 0.07^{\mathrm{a}}$ \\
& $\mathrm{S}$ & 150 & $9.36 \pm 0.07^{\mathrm{a}}$ \\
& $\mathrm{P}$ & 150 & $6.61 \pm 0.10^{\mathrm{a}}$ \\
& $\mathrm{S}$ & 250 & $7.12 \pm 0.10^{\mathrm{a}}$ \\
& $\mathrm{P}$ & 250 & $7.10 \pm 0.15^{\mathrm{a}}$ \\
& $\mathrm{S}$ & & $7.36 \pm 0.13^{\mathrm{a}}$ \\
& $\mathrm{P}, \mathrm{S}$ & & $9.98 \pm 0.12^{\mathrm{b}}$ \\
& $\mathrm{P}, \mathrm{S}$ & & $11.002 \pm 0.1^{\mathrm{c}}$ \\
& $\mathrm{P}, \mathrm{S}$ & & 0.000311 \\
\hline
\end{tabular}

\footnotetext{
a Calculated by comparative model.

b Calculated by model of Reintjes.

c Calculated by TDHF procedure.
}

or $\mathrm{Ga}$ atom lies out of $\mathrm{Pc}$ macrocycle plane and the $\mathrm{Cl}$ atom is in extra-coordination position, giving these molecules approximately $\mathrm{C}_{4 \mathrm{v}}$ symmetry. Additionally, the plane of the molecule is slightly domed (Fig.1).

In addition, GaClPc molecule is an example with a completely filled d-shell, where the metal ion is larger than the equilibrium cavity size of the phthalocyanine ring so giving larger cavity diameters. The structure of the molecule shows non-planarity and the gallium atom is $0.04 \AA$ out of the plane of the ring. The $3 \mathrm{~d}$ subshell of GaClPc is completely filled and deep enough to form rather pure molecular orbitals. This type of the molecular orbitals can give increasing for nonlinear optical properties. AlClPc molecule is an example, where the central metal atom also adopts position outside the Pc ring's plane. The $3 \mathrm{~d}$ subshell of AlClPc is not filled, which caused a more complex electronic structure. In this case, metal 3d levels lie between the HOMO and LUMO. For such systems, there are extra absorption features that may arise from
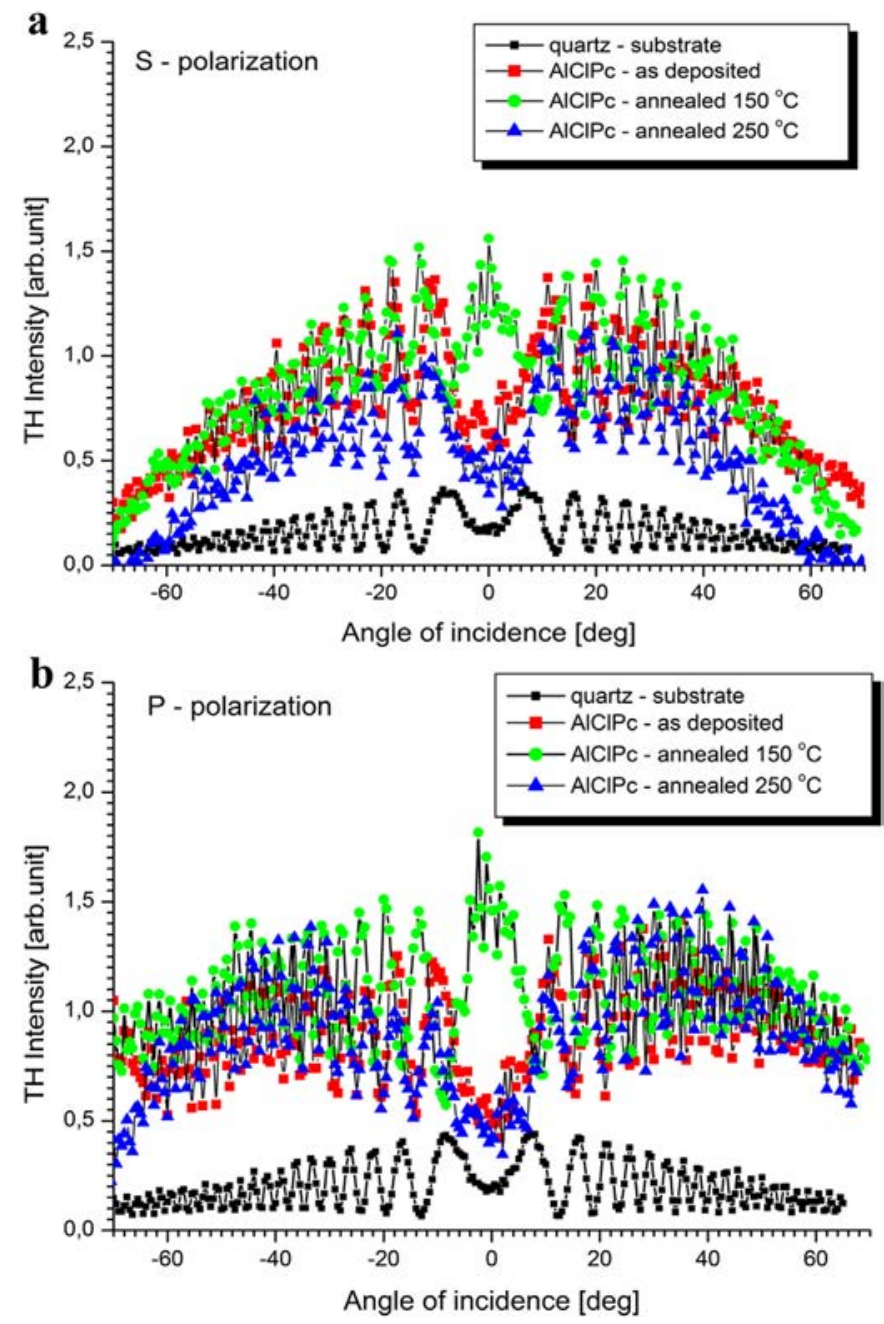

Fig. 7. THG spectra of AlCIPc: S - vertical (a) and P - horizontal (b) polarization of the laser radiation. 

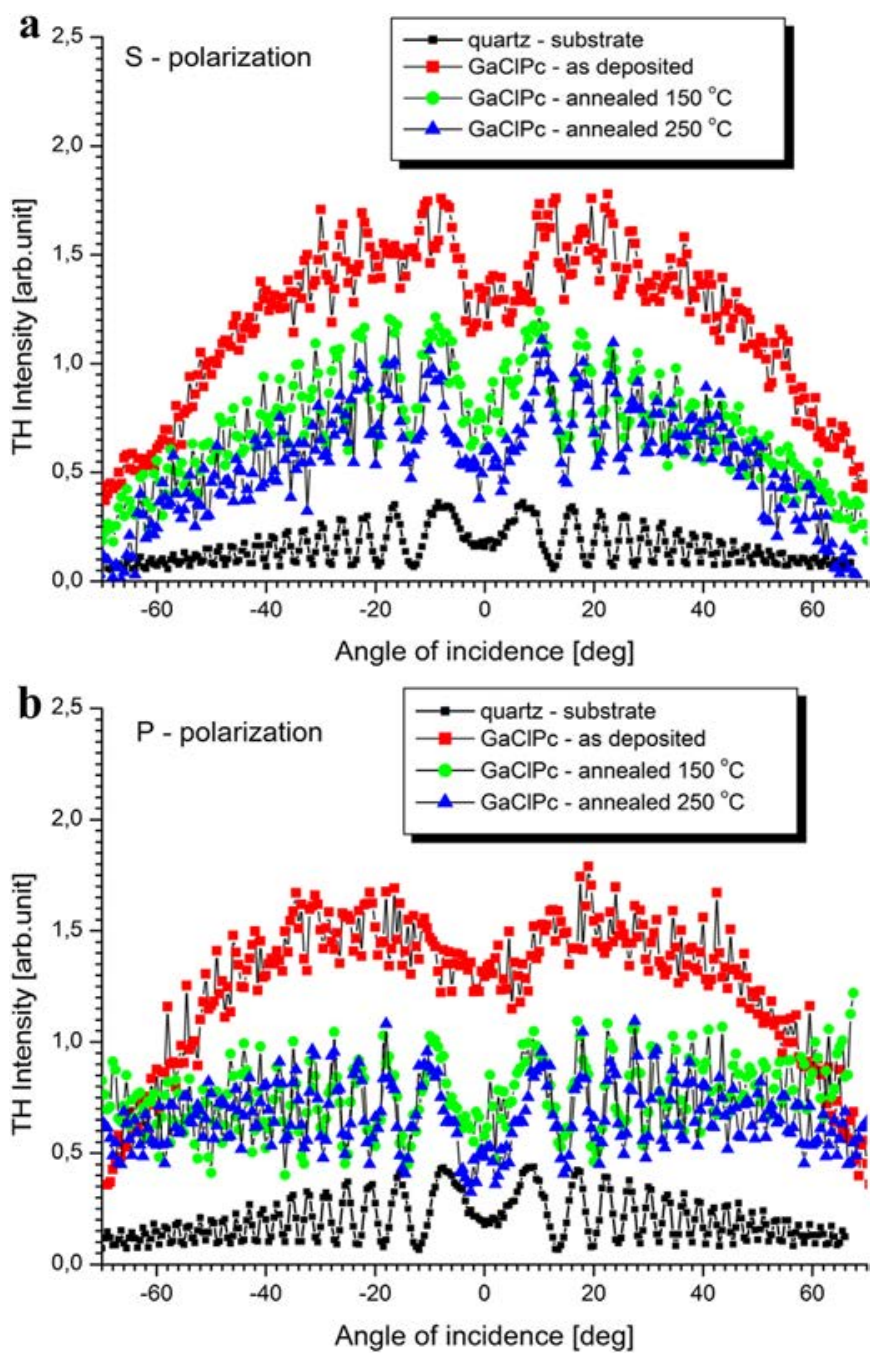

Fig. 8. THG spectra of GaClPc: S - vertical (a) and P - horizontal (b) polarization of the laser radiation.

charge transfer transitions. This type of the molecular orbitals can give decreasing of nonlinear optical properties. That is why the value of the real part of the third order nonlinear optical susceptibility $\chi_{\text {elec }}^{<3>}$ is slightly higher for GaClPc than AlClPc.

The presented THG experiment was performed at $1064 \mathrm{~nm}$ which gives a third harmonic at $355 \mathrm{~nm}$. The $355 \mathrm{~nm}$ lies closely at the B band in the absorption spectra of MClPcs (see Fig.4), which has a very strong absorption. Therefore, the large third order nonlinear susceptybility value of MClPc nanostructures may also be due to the three photon resonant contributions of the $B$ band. As a consequence of the three-photon resonance, the $\chi_{\text {elec }}^{<3>}$ value of MClPcs can be larger. The electronic contribution of $\chi_{\text {elec }}^{<3>}$ values for each studied compounds take into account the obtained values of the absorption coefficients. Comparing the measured and calculated susceptibilities of the MClPcs with the known values for fused silica glass (reference material), one can see four orders higher values of the nonlinear optical susceptibilities for all metallophthalocyanine chlorides (Table 9).

\section{Conclusions}

To reveal third order nonlinear optical properties of AlClPc and $\mathrm{GaClPc}$, THG experiments and theoretical calculation have been performed. This approach allowed us to determine the electronic contribution of the third order nonlinear optical susceptibility $\chi_{\text {elec }}^{<3>}$ of these MClPc films and to investigate three models for explanation of the observed results. Due to the assembly of the molecules, third order NLO properties were expected and could be more or less accurately predicted. For THG of nanostructural metallophthalocyanine chlorides thin films, it is difficult to give a complete rationalization of the factors governing the response. Moreover, the theoretical analysis is more complicated than that of SHG. However, to fully understand the third order optical nonlinearity behavior of AlClPc and GaClPc, we have performed detailed ab-initio quantum mechanical calculations to obtain the dispersion-free and frequencydependent dipole polarizabilities, second hyperpolarizabilities and third order susceptibilities. The transmission characterizations of the title molecules have been investigated both theoretically (CI method) and experimentally (UV-Vis spectroscopy). We have also examined the first and second frontier molecular orbitals of AlClPc and GaClPc molecules. The calculation results on the UV-Vis wavelengths and third order susceptibilities are quite consistent with the experimental values estimated in this work. Our computation results on third order susceptibilities are also in good agreement with the experimental results of similar metallophthalocyanine systems in the literature. An enhancement in the order AlClPc $<$ GaClPc on measured values of $\chi^{(3)}$ and computed values of $\chi^{(3)}$ and $\gamma$ shows the effect of the central metal in the metallophthalocyanine chloride structure on the third order NLO responses.

We finally found that the annealing process can significantly change the structural properties and cause a formation process of nanostructures inside thin films. Our experimental results and theoretical calculation clearly show that the value of the third order optical susceptibility makes this material interesting for future nonlinear optical applications.

\section{References}

[1] Marder SR, Gorman CB, Tiemann BG, Perry JW, Bourhill G, Mansou K. Relation between bond-length alternation and second electronic hyperpolarizability of conjugated organic molecules. Science 1993;261:186-9.

[2] Tackley DR, Dent G, Smith WE. Phthalocyanines : structure and vibrations. Phys Chem Chem Phys 2001;3:1419-26.

[3] Zawadzka A, Płóciennik P, Strzelecki J, Pranaitis M, Dabos-Seignon S, Sahraoui B. Structural and nonlinear optical properties of as-grown and annealed metallophthalocyanine thin films. Thin Solid Films 2013;545: 429-37.

[4] Mathews SJ, Kumar SC, Giribabu L, Rao SV. Nonlinear optical and optical limiting properties of phthalocyanines in solution and thin films of PMMA studied using a low power He-Ne laser. Mat Lett 2007;61:4426-31.

[5] Zawadzka A, Płóciennik P, Strzelecki J, Łukasiak Z, Sahraoui B. Photophysical properties of Alq3 thin films. Opt Mater 2013;36:91-7.

[6] Kambara H, Maruno T, Yamashita A, Matsumoto S, Hayashi T, Konami H, et al. Third-order nonlinear optical properties of phthalocyanine and fullerene. J Appl Phys 1996;80:3674-82.

[7] Sanghadasa M, Wu B, Clark RD, Guo H, Penn BG. Third order nonlinear optical properties of metal phthalocyanines with butoxy and decyloxy substituents. Proc SPIEs Int Soc Opt Eng 1997:3147:185-95.

[8] Nalwa HS, Kobayashi S. Large third order optical non-linearities of spin-cast thin films of novel metallo-naphthalocyanines. J Porphyrins Phthalocyanines 1998:2:21-30.

[9] Kajzar F, Messier J, Rosilio C. Nonlinear optical properties of thin films of polysilane. J Appl Phys 1986;60:3040-4.

[10] Sahraoui B, Rivoire G, Terkia-Derdra N, Sallé M, Zaremba J. Third order nonlinear optical properties of new bisdithiafulvenyl-substituted tetrathiafulvalene. JOSA B 1998;15:923-8.

[11] Rau I, Kajzar F, Luc J, Sahraoui B, Boudebs G. Comparison of Z-scan and THG derived nonlinear index of refraction in selected organic solvents. JOSA B 2008;25:1738-47.

[12] Zawadzka A, Płóciennik P, Czarnecka I, Sztupecka J, Łukasiak Z. The effects of annealing process influence on optical properties and the molecular orientation of selected organometallic compounds thin films. Opt Mater 2012;34: 1686-91.

[13] Zieliński M, Dygdała RS, Karasek K, Zawadzka A. Real-time multichannel scaler measurement of oscillator instabilities. Rev Sci Instrum 2000;71: 2577-81.

[14] Zawadzka A, Płóciennik P, Strzelecki J, Łukasiak Z, Bartkiewicz K, Korcala A, et al. Optical properties of metallophthalocyanine compounds thin films. 
Transparent optical networks (ICTON). In: 14th international conference; 2012. http://dx.doi.org/10.1109/ICTON.2012.6253720.

[15] Zawadzka A, Dygdala RS, Raczynski A, Zaremba J, Kobus J. Three-photon resonances due to autoionizing states in calcium. J Phys B Mol Phys 2002;35: $1801-17$.

[16] Dygdała RS, Zieliński M, Płóciennik P, Zawadzka A, Rumianowski R. Time-offlight repeller circuit application in laser ablation experiment. Surf Coat Technol 2009;203:2328-32.

[17] Maker PD, Terhune RW, Nisenhoff M, Savage CM. Effects of dispersion and focusing on the production of optical harmonics. Phys Rev Lett 1962;8:21-2.

[18] Zawadzka A, Płóciennik P, Strzelecki J, Korcala A, Arof AK, Sahrauoi B. Impact of annealing process on stacking orientations and second order nonlinear optical properties of metallophthalocyanine thin films and nanostructures. Dyes Pigment 2014;101:212-20.

[19] Dykstra CE. Dipole (electric field) and quadrupole (field gradient) polarizabilities of hydrogen, nitrogen, and acetylene from the application of derivative Hartree-Fock theory. J Chem Phys 1985;82:4120-5.

[20] Liu S, Dykstra CE. Multipole polarizabilities and hyperpolarizabilities of AHn and A2Hn molecules from derivative Hartree-Fock theory. J Phys Chem 1987;91:1749-54.

[21] Maroulis G. On the accurate theoretical determination of the static hyperpolarizability of trans-butadiene. J Chem Phys 1999;111:583-91.

[22] Shelton DP, Rice JE. Measurements and calculations of the hyperpolarizabilities of atoms and molecules in the gas phase. Chem Rev 1994;94: $3-29$.

[23] Intel $\times 86$ (win32, Linux, OS/2, DOS) version. PC GAMESS version 6.2, build number 2068. This version of GAMESS is described in: Schmidt MW, Baldridge KK, Boatz JA, Elbert ST, Gordon MS, Jensen JJ, et al. General atomic and molecular electronic structure system J Comput Chem 1993;14:1347-63.

[24] Frisch MJ, Trucks GW, Schlegel HB, Scuseria GE, Robb MA, Cheeseman JR, et al. Gaussian 03, revision E.01. Wallingford CT: Gaussian, Inc.; 2004.

[25] Bredas JL, Adant C, Tackx P, Persoons A, Pierce BM. Third order nonlinear optical response in organic materials: theoretical and experimental aspects. Chem Rev 1994;94:243-78.

[26] Mongwaketsi N, Khamlich S, Pranaitis M, Sahraoui B, Khammar F, Garab G, et al. Physical origin of third order non-linear optical response of porphyrin nanorods. Mater Chem Phys 2012:13:4646-50.

[27] Lee GJ, Cha SW, Leon SJ, Jin JI, Yoon JS. Second order nonlinear properties of unpoled bent molecules in powder and in vacuum deposited film. J Korean Phys Soc 2001;39:912-5.
[28] Reintjes JF. Nonlinear optical parametric processes in liquids and gases Quantum electronics, Principles and applications. Academic Press Inc.; 1984

[29] Cullity BD. Elements of X-ray diffractions. Reading, MA: Addison Wesley; 1978.

[30] Iwatsu F, Kohayashi T, Uyeda N. Solvent effects on crystal growth and transformation of zinc phthalocyanine. J Phys Chem 1980;84:3223-30.

[31] Mathew S, Sudarsanakumar C, Menon CS. Optical, electrical and structural characterization of gallium phthalocyanine chloride thin films. Optoelectron Adv Mater - Rapid Commun 2007;1:614-9.

[32] Ortí E, Bredas JL, Clarisse C. Electronic structure of phthalocyanines: theoretical investigation of the optical properties of phthalocyanine monomers dimers and crystals. J Chem Phys 1990;92:1228-35.

[33] Lu YT, Ge JF, Xu QF, Yang Y, Li NJ, Sun R, et al. Third order nonlinear optical properties of the phenothiazinium chlorides at $532 \mathrm{~nm}$. Dyes Pigment 2011;89:70-5.

[34] Kadish KM, Smith KM, Guilard R. The porphyrin handbook: phthalocyanines properties and materials. San Diego: Academic Press; 2003. Elsevier Science.

[35] Sen BN, Mert H, Dincer $H$, Koca A. Synthesis and characterization of terminalalkynyl-substituted unsymmetrical zinc phthalocyanine conjugated with well-defined polymers. Dyes Pigment 2014;100:1-10.

[36] Klofta TJ, Rieke PC, Linkous CA, Buttner WJ, Nanthakumar A, Mewborn TD, et al. Tri- and tetravalent phthalocyanine thin film photoelectrodes: comparison with other metal and demetallated phthalocyanine systems. Electrochem. Soc 1985;132:2134-44.

[37] Van Gisbergen SJA, Snijders JG, Baerends EJ. Accurate density functional calculations on frequency-dependent hyperpolarizabilities of small molecules. J Chem Phys 1998;109:10657-68.

[38] Sutherland RL. Handbook of nonlinear optics. New York: Marcel Dekker Inc.; 1996.

[39] Díaz-García MA, Ledoux I, Duro JA, Torres T, Agulló-López F, Zyss J. Third order nonlinear optical properties of soluble octasubstituted metallophthalocyanines. J Phys Chem 1994;98:8761-4.

[40] Sastre A, Díaz-García MA, del Rey B, Dhenaut C, Zyss J, Ledoux I, et al. Pushpull phthalocyanines: a Hammett correlation between the cubic hyperpolarizability and the donor-acceptor character of the substituents. J Phys Chem 1997;101:9773-7.

[41] Sun W, Wang H, Sun J, Kan J, Chen Y, Jiang J. 2,3,9,10,16,17,23,24-Octaki$\mathrm{s}$ (phenoxy/octyloxy)phthalocyaninato manganese complexes. Synthesis, structure, and nonlinear optical property. Dyes Pigment 2013;99:154-9.

[42] Nalwa HS, Kakuta A, Mukoh A. Third order nonlinear optical properties of a vanadyl naphthalocyanine derivative. J Phys Chem 1993;97:1097-100. 